\title{
INTEGRATION OF PUBLIC TRANSPORT THROUGH A STRUCTURED ANALYSIS TECHNIQUE
}

\author{
D. ALLOPI \\ Department of Civil Engineering and Surveying, Durban University of Technology, Durban, South Africa.
}

\begin{abstract}
One of the strategic objectives of the present government of South Africa is to promote the use of public transport with a goal of achieving a ratio of 80:20 between public transport and private car usage by the year 2020. However, the present structures of most cities are not conducive to the development of efficient public transport systems. Occasionally, expensive infrastructures were constructed in certain areas without proper planning. Chatsworth, a major suburban area of Durban, South Africa, has been considered as a case study in this article. The area is connected to the city centre by public transport systems, namely bus, minibus and metro rail. Surprisingly, the patronage on the rail system is very low and is decreasing. With a view to increase the accessibility to the rail system, a methodology has been presented based on data flow diagrams to develop a computer simulation model for the operational analysis of minibus taxi-rail integration. The service requirements of metro rail and the minibus taxi service were analysed based on different demand scenarios in order to determine the optimum service requirement for the integrated system.

Keywords: Chatsworth, commuter, data flow diagrams, Durban, metro rail, minibus taxi, prototype, transport infrastructure.
\end{abstract}

\section{INTRODUCTION}

The transportation sector is a major source of pollution, especially of the air with motor vehicles being the main contributory factor. Air pollution from vehicles has adverse effects on human health, plant and animal life and further cause property damage (soiling and deterioration of building surfaces). Rail transport is often advocated as an alternative that will make the air cleaner [1].

Macpherson [2] emphasised that transport was one of the major causes of environmental crises. Virtually all vehicles burn fossil fuels, either directly or indirectly and it is clear that a reduction in the amount of noxious substances from road vehicles would lead to a significant improvement in environmental quality. Most governments in developed countries accept that environmental issues should be accorded a high priority.

In recent years, the emphasis on economic efficiency and the optimum utilisation of existing transport infrastructures have featured high on the governments' agendas universally. South Africa is no exception and is currently seeking strategies to optimise capacity utilisation and to achieve a level of integration between modes to achieve a ratio of 80:20 between public transport and private car usage by 2020 as targeted in the White Paper on National Transport Policy [3]. In the past, a fragmented approach was taken in transportation planning. As a result, expensive infrastructure was constructed in certain areas without proper planning.

Chatsworth, one of the major suburban areas in the city of Durban, is a typical example of such planning. It is situated approximately $26 \mathrm{~km}$ from the central business district (CBD) with a population in excess of 200000 and car ownership of about 40000 , according to the 1996 census. The vehicles on Higginson Highway (M1), the only major access road from this area to the Durban CBD, operate at capacity during peak hours. Volumes in excess of 4800 vehicles per hour have been recorded during the morning peaks [4]. This has resulted in the public demanding for the construction of a second access road to the area. Chatsworth is also connected to the CBD by a surface rail system which is operated by metro rail. There are five rail stations in the area, namely, Havenside, Bayview, Westcliff, Chatsglen and Crossmoor. 
Since the area is inhabited by people of different income groups, a substantial percentage of the commuters still rely on public transport. Surprisingly, the patronage on the rail service is extremely low when compared with bus and minibuses and is decreasing gradually.

The status quo report [5] indicates a potential growth in the utilisation of $265 \%$ on the DurbanChatsworth rail line. This is a unique case of underutilisation of existing infrastructure when, on the one hand, there is a demand to construct a new access road and on the other hand, an expensive rail system remains almost unused.

Improper land-use planning and the alignment of the rail in relation to the residential dwellings are two important reasons for the decline of the rail system in Chatsworth. It has been estimated that less than $5 \%$ of the residents live within $500 \mathrm{~m}$ of a railway station, highlighting the lack of accessibility [6]. Also, the parking facilities at the stations are inadequate and unsafe which discourage the commuters from 'park and ride'. Moreover, bus and minibus services from the residential areas to the railway stations are inadequate.

A study was carried out to establish deficiencies in the existing transportation infrastructure and services and the response of the commuters revealed that the distance to the rail station and inadequate safety were the primary causes for the underutilisation of the metro rail system [7]. The study identified the lack of access, lack of convenient ticket sales offices, restricted hours of ticket sales, inadequate and poor facilities/infrastructure, and limited service as the other contributing factors. With regard to the commuter survey, it was interesting to note that $80 \%$ of the present car users indicated that they would prefer public transport if the existing conditions were improved [8]. The factors that they considered most important to be improved on the rail service included: security while travelling, improved frequency of rail and minibus service from their locality to the main station. It was quite evident from the analysis that the present rail users showed greater discontent with the level of service in comparison with bus and minibus commuters.

To encourage commercial and residential development along the rail corridor is one of the longterm solutions to increase accessibility. However, in the short run, the integration of the rail system with the road-based modes may be the only possible solution to increase the utility of the rail service in Chatsworth. This article proposes a methodology based on data flow diagrams to integrate the rail system with the minibus taxi services [9].

\section{DEMAND ANALYSIS}

One of the focuses of this case study was to also demonstrate the sensitivity of the integrated system proposed with regard to population, employment, capacity, frequency of service and the various other elements that may have an impact on the system. The level of demand during the peak period was used to calculate the peak fleet requirement.

One should be able to distinguish between the capacity of the road network and the capacity of the transit system. The capacity of a road is its ability to carry traffic and is measured by the maximum number of vehicles that may pass a given point of a lane of the road in one direction during a specified time (usually taken as one hour). Road capacity may be better appreciated through an analysis of the relationships between the speed, flow and density of traffic. The capacity of a transit system is usually measured by the maximum number of persons that can be moved on a single track or single lane in one hour. It is important to determine whether a line can handle peak hour demand.

\subsection{Employment demand}

Assuming a $10 \%$ growth on the 1996 census figures, the population for the greater Chatsworth area would be approximately 220000 . The home interview survey conducted in the area revealed an 
average household size of six members with an average of approximately two employed persons per household. Based on the above, the number of employed persons in the survey area would be approximately 73400 .

\subsection{Vehicle capacity}

The capacity of a taxi/train route is the product of the passenger capacity per taxi or train and the maximum number of taxis or trains that can travel on the route during a specified period of time.

According to the Chatsworth Minibus Association, approximately 300 taxis operate on the inner circle route (i.e. in the greater Chatsworth area) with a smaller proportion allocated to the Durban CBD route. For the purpose of this study, the Toyota Hiace (Super 16) was chosen as the representative vehicle, as it was found that a higher percentage of the taxi operators used this vehicle. This minibus has a Toyota $4 \mathrm{Y}$ engine (capacity of $2237 \mathrm{~cm}^{3}$ ), $2495 \mathrm{~mm}$ wheelbase, an overall length of $4725 \mathrm{~mm}$ and a gross vehicular mass of $2650 \mathrm{~kg}$. Based on a maximum of 300 taxis engaged in the integrated system, the total number of passengers per trip transported to the stations will be 4800 (based on a capacity of 16 passengers per taxi).

The capacity of rail transit may be expressed as the maximum number of passengers that may be carried by the system in one hour per track in one direction. The capacity is given by:

$$
\mathrm{Cm}=60 \mathrm{~W} / \mathrm{hf},
$$

where $\mathrm{Cm}=$ capacity expressed as number of passengers per track in one hour in one direction; $W=$ crush load, i.e. the maximum number of passengers per train during peak hour; $h f=$ headway in frequency of train movement along the track per minute.

$W$ depends upon the size of each coach and the number of coaches per train. The number and composition of made up train sets operating on the Durban-Crossmoor line are shown in Table 1.

The passenger capacity per train set on the Durban-Crossmoor line $=1210$ (normal) and 1515 (crush load), where the crush load is equal to the normal load plus approximately $25 \%$ [5].

There is considerable potential to increase the frequency of service provided on this line. The scheduled peak hour service on the Durban-Crossmoor line is made up of three trains with a passenger capacity of 4545 [5]. The potential line capacity is equivalent to 11 trains with a passenger capacity of 16665 at peak hour yielding a potential growth in capacity of approximately $265 \%$. Table 2 shows

Table 1: Train set make-up: Durban-Crossmoor service [5].

\begin{tabular}{ll}
\hline Number of train sets & 5 \\
Motor coaches & 1 \\
First class & 2 \\
Third class & \\
Plain trailers & 1 \\
First class & 5 \\
Third class & 9 \\
Total number of coaches &
\end{tabular}

Motors and trailers are either first or third class, the difference being in terms of the number and quality of seating provided as well as the standard of lighting. 
Table 2: Varying peak hour service versus passenger capacity.

\begin{tabular}{cc}
\hline Number of trains (peak hour) & Passenger capacity \\
\hline 4 & 6060 \\
5 & 7575 \\
6 & 9090 \\
7 & 10605 \\
8 & 12120 \\
9 & 13635 \\
10 & 15150 \\
\hline
\end{tabular}

Table 3: Main modes of transport to work.

\begin{tabular}{lc}
\hline Mode & Percentage \\
\hline Car & 52.3 \\
Bus & 31.4 \\
Train & 4.6 \\
Taxi & 11.7 \\
\hline
\end{tabular}

the passenger capacity for the peak hour services based on 'crush' loads. It could also be possible to increase the size of the train sets to 14 coach sets, the capacity of the service provided could be increased by approximately $55 \%$.

\subsection{Integrated system demand}

The main modes of transport to work as determined from the commuter survey conducted in the area are shown in Table 3.

It was also interesting to note that $80 \%$ of the present car users indicated that they would consider public transport if the existing conditions were improved. Based on the assumption that only $10 \%$ of the present car users, as well as taxi and bus users will opt for the integrated system, the percentage demand will be $0.1 \times 52.3+0.1 \times 31.4+0.1 \times 11.7+4.6$ (no shift in present rail patronage), i.e. just under $15 \%$ of the employment force will be absorbed by this system yielding approximately 11000 commuters that will need to be accommodated. Based on 300 minibuses allocated to the integrated system, all 300 taxis will be required to make two trips to the designated stations with approximately 88 taxis having to make an additional (third) trip. The number of trains required during the peak hour will be eight at headway of approximately 7.5 minutes (based on a crush load capacity of 1515).

Table 4 shows the total demand on both the public transport modes assigned to the integrated system (IS) during peak hour.

As previously mentioned, the maximum number of passengers per train during peak hour depends upon the size of each coach and the number of coaches per train. The headway will depend on the signalling system, speed of train, acceleration and deceleration rates, length of train, station stop time, and so on. 
Table 4: Demand analysis of the integrated system.

\begin{tabular}{rcccc}
\hline IS demand $(\%)$ & $\begin{array}{c}\text { No. of } \\
\text { commuters }\end{array}$ & $\begin{array}{c}\text { No. of trips (no. of } \\
\text { Taxis) }\end{array}$ & No. of trains & $\begin{array}{c}\text { Headway, } \\
\text { min. (rail) }\end{array}$ \\
\hline 5 & 3670 & $1(230)$ & 3 & 20 \\
10 & 7340 & $1(300) ; \mathrm{A}(159)$ & 5 & 12 \\
15 & 11010 & $2(300) ; \mathrm{A}(88)$ & 8 & 7.5 \\
20 & 14680 & $3(300) ; \mathrm{A}(18)$ & 10 & 6 \\
\hline
\end{tabular}

$\mathrm{A}=$ Additional trip.

Table 5: Demand analysis of metro rail (14 coach train set).

\begin{tabular}{lc}
\hline IS demand $(\%)$ & $\begin{array}{c}\text { Number of trains (peak } \\
\text { hour) }\end{array}$ \\
\hline 5 & 2 \\
10 & 4 \\
15 & 5 \\
20 & 7 \\
\hline
\end{tabular}

Capacity may be increased by increasing the maximum number of passengers per train during the peak hour and/or decreasing the headway. To increase the crush load capacity, the length of the train has to be increased but then the headway shall increase unless the speed is also increased. If we look at the option of increasing the size of the train sets to 14 coach sets, which is possible, the crush load passenger capacity can be increased to 2350 . Table 5 shows the impact this will have on the number of trains required during the peak hour based on the projected demands, assuming that the headway remained the same. This would obviously require increasing the speed.

It can be observed from Tables 4 and 5 that the reduction in the number of trains required during peak hour is more significant as the demand increases.

It must be noted that the above exercise demonstrates the overall or total demand on the proposed system. A pilot study carried out in the survey area will assist in determining the demand on individual taxi routes. This would help the minibus taxi association to distribute their fleet proportionately. It is also evident that there is considerable potential to increase the capacity of the rail line when compared with the current scheduled service.

\section{DEVELOPMENT OF AN INTEGRATED SYSTEM}

It was found that the minibus association operated on the inner circle route (greater Chatsworth area) and recently allocated a small percentage of its fleet to the Durban CBD route which resulted in conflict with the bus association. Interviews conducted by the author with the Chatsworth Minibus Association with regard to the formation of a partnership with rail to provide a feeder service to the stations proved promising and positive. The South African Rail Commuter Corporation (the authority responsible for train services in South Africa) supports an integrated public transport system. It was therefore decided to look closely at this option and to formulate an integrated system between the 
minibus association and rail services. A methodology has been presented for developing a computer simulation model for the operational analysis of minibus and rail integration.

The primary aim is to set up an integrated transport system in the survey area to capture commuters who will eventually become 'committed' to the system. Integrated fares would be introduced and made attractive (greater discount) for the purchase of a monthly, semester, or even an annual transport card. To implement the integrated system, the server should be set up at the Chatsworth Centre (shopping complex), where the bulk of the residents from the area make their purchases and account payments. Terminals would be located at five railway stations and at some of the post office depots in the area, with a view of introducing automatic ticket dispensers at a later stage. Card readers/validation machines will have to be installed on minibuses and at the entrance to the station platforms. Commuters will be required to validate their cards at the commencement of the trip. Enforcement and appropriate fines will have to be imposed in order to prevent fare evasion.

Through a structured analysis technique called data flow diagrams (DFDs), a graphical representation of data processes throughout the organisation for an integrated system is presented. DFDs indicate the flow and transformation of data within a system [10]. They serve as a strong communication vehicle and this approach emphasises the logic underlying the system. Using combinations of only four basic components, one can create a pictorial depiction of processes that will eventually provide solid system documentation.

\subsection{Components of a DFD}

A DFD consists of four basic components, namely external entities, processes, data flows, and data stores. The characteristics and use of each of these components is reviewed below.

\subsubsection{External entities}

They are entities which are outside the system but which communicate with the system. In this case, the typical external entities will be the commuters, minibus association, metro rail, and accounts. These entities can send data to or receive data from the system. A rectangle indicates any entity external to the system being modelled.

\subsubsection{Processes}

Processes always denote a change in or transformation of data. They represent work being performed within the system. Processes should be cohesive and have as few inputs and outputs as possible. An example of a process will be to update the route schedule for minibuses. Bubbles or circles are used to indicate the points within the system at which incoming data flows are processed or transformed into outgoing data flows.

\subsubsection{Data flows}

Arrows denote movement of data from one point to another, with the head of the arrow pointing towards the data's destination. In simple terms, data flows describe data that flows through the system. Data flows should include the minimum essential data needed by the process that receives the data flow. All data flows should begin and/or end at a process, because data flows either initiate a process or result from a process.

\subsubsection{Data stores}

They are passive stores of information. The type of physical storage (tape, diskette, etc.) is not specified. At this point, the data store symbol simply shows a depository for data that allows addition 
and retrieval of data. A data flow from a data store to a process means that the process uses the data while a data flow to a data store means that the process updates (adds, deletes or changes) the data store. Open rectangles are used to identify temporary holding points for collection of data.

\subsection{Developing the DFD}

To begin a DFD, the organisation's system narrative is collapsed into a list with the four categories: external entity, data flow, process and data store. This list in turn helps determine the boundaries of the system to be described. The first is to draw a context diagram, followed by the top level diagram and then the detail level DFD. These are discussed very briefly below.

\subsubsection{Context diagram}

The context diagram gives an overview of the systems interaction with its environment. The environment consists of the external entities and data stores that are considered to be outside the system. The context diagram is the highest level in a DFD and contains only one process, representing the entire system. A typical context diagram for an integrated public transport system as developed in this study involving rail and minibus is shown in Fig. 1.

The external entities such as minibus association, metro rail, the commuter and accounts are shown in this diagram and the major data flows to and from the external entities are also shown. The context diagram contains only one process (Integrated Transport System) that represents the entire system.

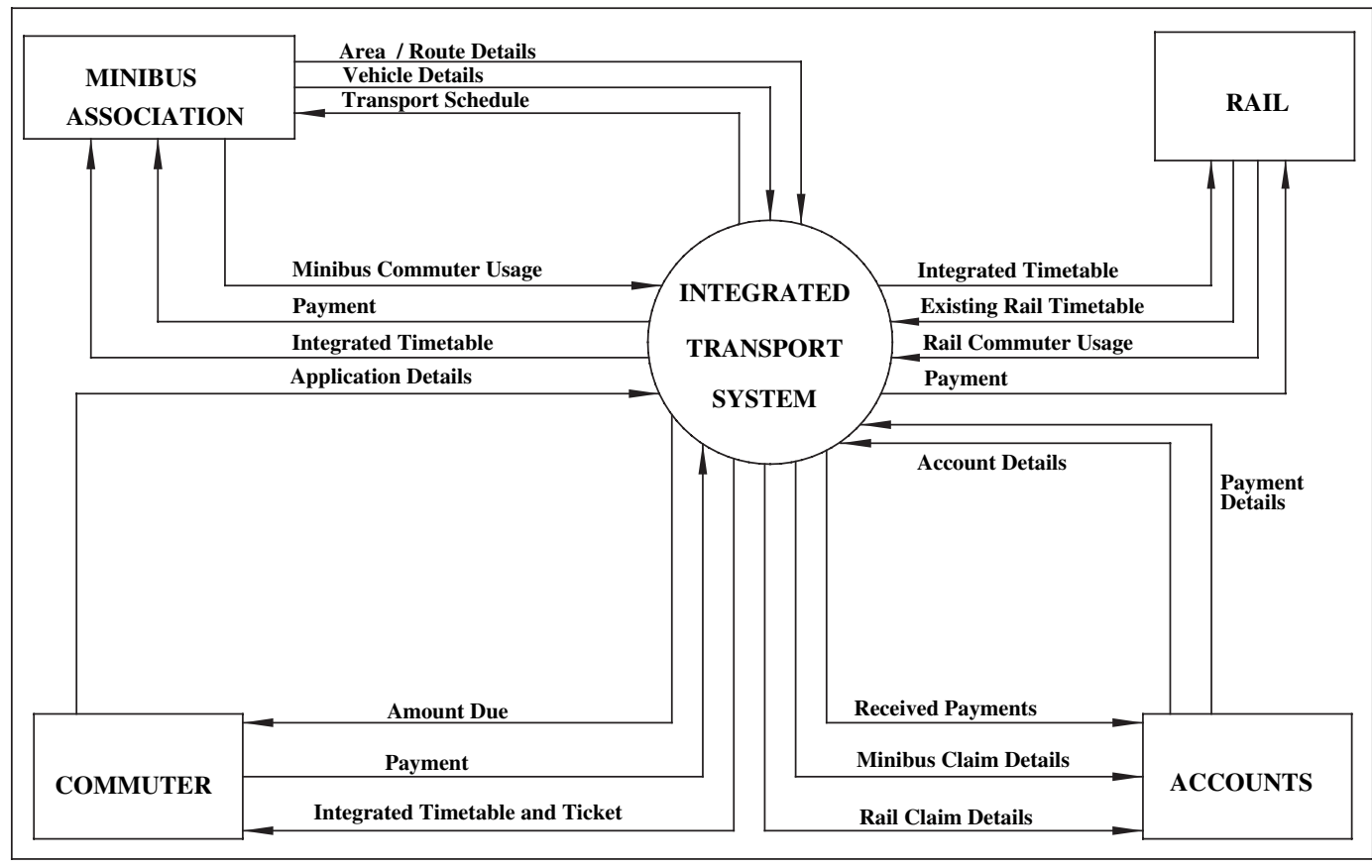

Figure 1: Context Diagram for the Integrated Transport System. 


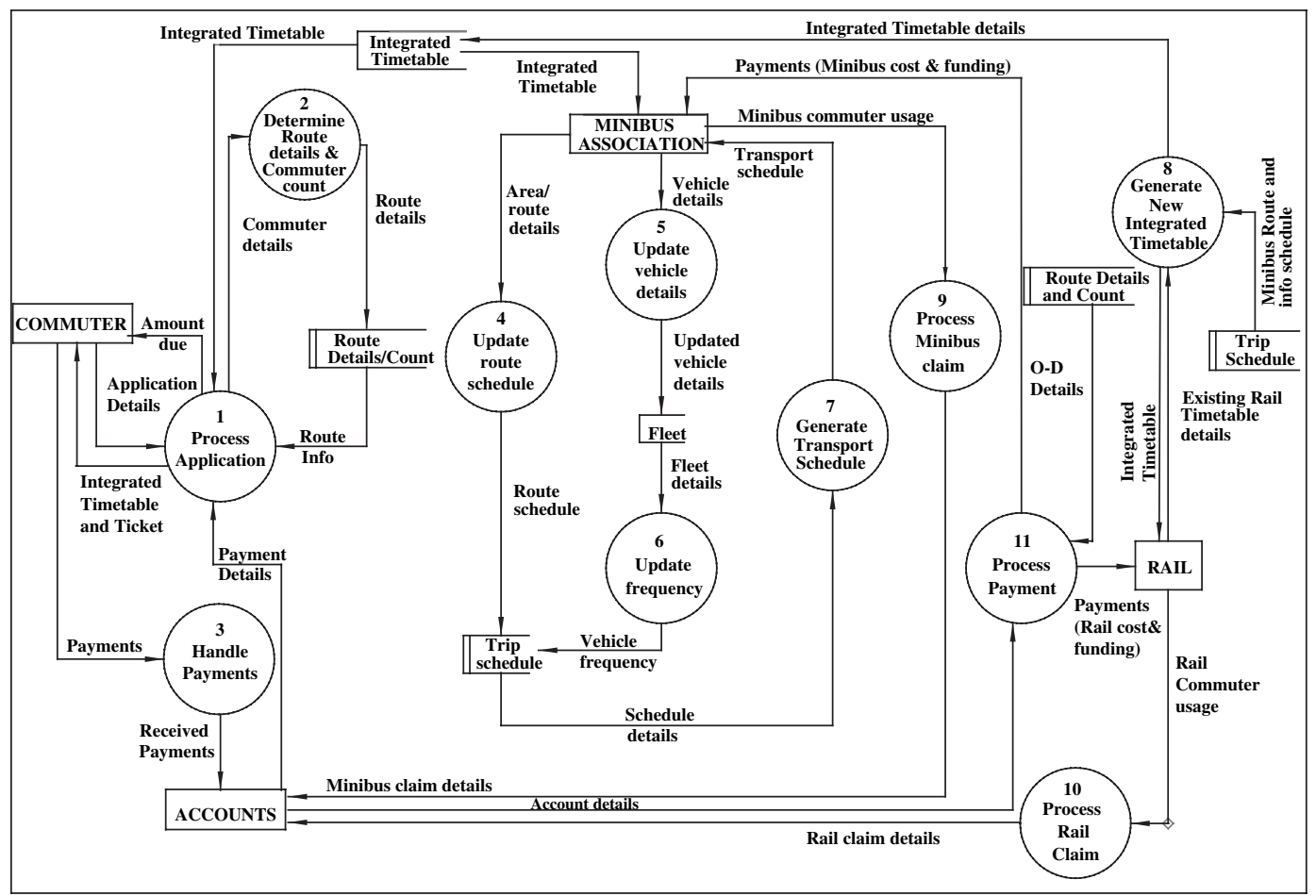

Figure 2: Top-level DFD for the integrated system.

\subsubsection{Top-level DFD (diagram 0)}

This diagram gives an overview of the main functions of the system and the flow of data through the system. Each main function is represented by only one process. Diagram 0 is an explosion of the context diagram and may include up to 11 processes. This diagram still contains all the same inputs and outputs as the context diagram, except that the single central bubble in the context diagram has been partitioned or decomposed into a series of components.

The top-level DFD (diagram 0) is shown in Fig. 2.

This diagram gives an overview of the main functions of the system and the flow of data through the system. Each main function is represented by only one process. Diagram 0 is an explosion of the context diagram and may include up to 11 processes. This diagram still contains all the same inputs and outputs as the context diagram. The only difference is that the single central bubble in the context diagram has been partitioned or decomposed into a series of components. The major data stores of the system (Route Details/Count, Trip Schedule, Fleet, Integrated Timetable) and all external entities (Commuter, Metro Rail, Taxi Association, Accounts) are included in diagram 0. Since a DFD is two-dimensional (rather than linear), it is possible to start at any point and work forward or backward through the diagram. The COMMUTER (external entity) requests to use the system indicating his/her origin and destination as part of the application details required to process the application (Process 1). The physical address as part of commuter details will assist in identifying the route node closest to the commuter as reflected in Process 2. Route details pertaining to all commuter requests are stored in a database called 'ROUTE DETAILS/COUNT'. The direction of arrows relating to data stores is important since data flow from a data store to a process means that the process uses the data, 
i.e. a 'read' is assumed. On the other hand, a data flow to a data store means that the process updates (adds, deletes or changes records) the data store. Based on a radius system for the determination of fares, the amount due is received from the commuter. All payments are deposited in a central accounting system called ACCOUNTS (external entity) via the handle payments process (Process 3 ). The TAXI ASSOCIATION (external entity) supplies a transport schedule based on the designated routes and nodes through the various units in the area to one of the five railway stations closest to that particular unit. The system allows for updating route schedule (Process 4) depending on commuter demand. Information pertaining to route details is placed in the data store TRIP SCHEDULE. The schedule also takes into account the available fleet of the association in determining the frequency of the service to be provided. Once again, depending on passenger demand, the vehicle details may be updated (Process 5) with FLEET being assigned as the data store. Fleet details are utilised in updating the frequency of service to be provided (Process 6). Vehicle frequency details are also stored in TRIP SCHEDULE. The taxi schedule details (route schedule and vehicle frequency) are used to generate a transport schedule (Process 7) for the taxi industry. The taxi transport schedule is then combined with the existing timetable for METRO RAIL (external entity) to generate a new integrated time-table (Process 8), copies of which are directed to both the public transporters. It is obvious that adjustments would have to be made from time-to-time to either or both of the public transporters' timetable depending on the demand for the integrated transport system (ITS). All commuters that have paid (via the application process) to use the system receive a copy of the integrated timetable together with his/her computerised ticket/travel card.

Depending on the taxi commuter usage, the amount due to the Taxi Association is determined (Process 9) and the taxi claim details are forwarded to ACCOUNTS (external entity). Similarly, based on the rail commuter usage, the amount due to Metro Rail is calculated (Process 10) and also forwarded to ACCOUNTS. Account details are verified against commuter details initially placed in the data store ROUTE DETAILS/COUNT. The final payment (Process 11) is based on the assumption that some funding/subsidy is made available by government in order to keep the integrated fare as low as possible thus attempting to make the system more attractive to commuters and further assisting with the integrated system gaining momentum.

\subsubsection{The detailed level DFD (child diagram)}

Each process in diagram 0 may in turn be exploded to create a more detailed child diagram. However, it should be noted that processes may or may not be exploded, depending on their level of complexity. It is always advisable to use original, unexploded data flow diagrams (as in Fig. 2) in the early stage when ascertaining information requirements.

Overly exploded diagrams may not be helpful to the users, since changes will need to be incorporated after getting users input.

Data flow balancing is necessary where flows are carried over from a higher level to a lower level. On this level, data stores which are local to a process can be shown for the first time. All data flows in or out of the parent process must be shown flowing in or out of the child diagram.

The numbering of the child diagram is derived from the numbering of the parent process in the top-level data flow diagram (diagram 0 ).

For the purpose of illustration, an example of the detail level DFDs is shown in Fig. 3. Figure 3 shows the application process where the commuter requests to use the facility indicating the origin and destination (Process 1.1). This information, obtained from all commuters, is stored in a database for calculating the amount due by the commuter, as well as for the purpose of audit/payment to the taxi association and metro rail. In determining the amount due by the commuter, the following two approaches may be considered. First, the radius may be determined from the route node closest to 


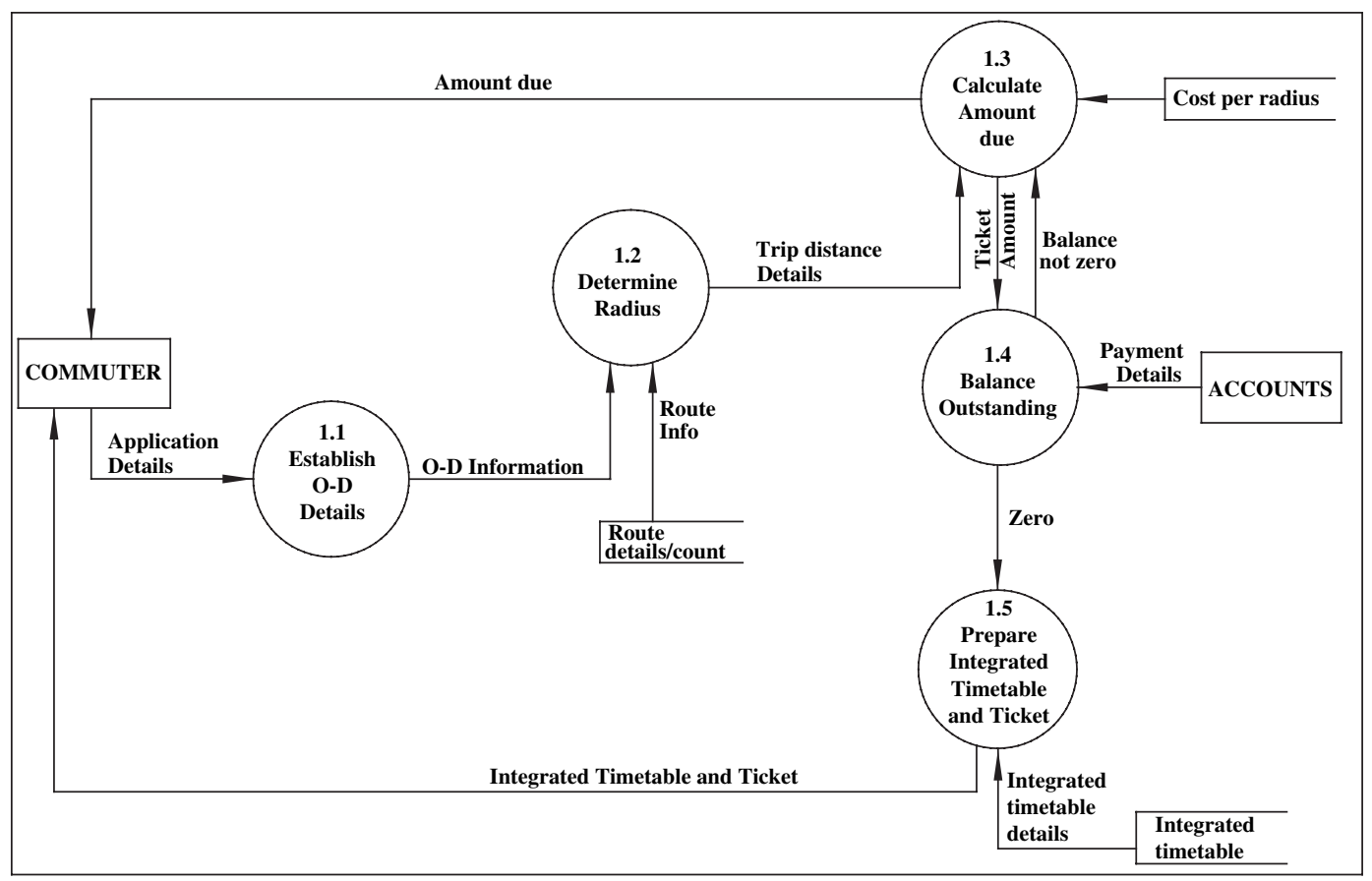

Figure 3: Detail level DFD: application process.

the locality of the commuter to the destination rail station (Process 1.2). The cost factor based on the radius system or a flat rate may then be applied to determine the amount payable (Process 1.3).

This cost added to the fixed minibus fare will yield the total amount due by the commuter (Process 1.3). On payment of the amount due, the commuter is given an integrated timetable together with the ticket/travel card. The system could be designed to check for outstanding payments, as in the example and as reflected in Process 1.4.

\section{DEVELOPMENT OF A PROTOTYPE}

Considering the human and financial resources available and the constraints on time, it was not possible to go for a full-scale model. It was therefore decided to develop a prototype model following the methodology based on DFDs. Prototyping can be classified as a modelling approach which emphasises the creation of abstract description of a software system to answer questions about the system. The emphasis is on the determination of the feasibility of a proposed solution before investing in the large-scale development of the target system. The intention is to go a step closer to the pilot program by developing a prototype model program. The prototype system fulfils the most important requirements of the user in an undetailed way, in a relatively short time using tools, such as screen generators. The user will be able to determine from the input and output screens as to whether his or her needs are being met by the software thus avoiding expensive programming.

The prototype was developed around the option of using the integrated public transport system (minibus and rail), since this was the main purpose or focus of the exercise. A range of ticket options ranging from a single to a yearly ticket were included in the input screen. For the purpose of demonstration, the first three options were included in the prototype (single, return, and weekly ticket). 
The default was set on Bayview station as the origin/boarding station. The destination stations were set on Merebank, Clairwood and Durban for the single, return, and weekly ticket options, respectively. With regard to the taxi route, three nodes on Pelican Drive (Pelican/Turnstone, Pelican/Skylark, Pelican/Liberty) were considered as typical stops leading to Bayview Station. The system was designed on the assumption that both the taxis and trains strictly follow the time schedule. However, a buffers of 10 minutes was allowed between the arrival time of the taxis at the station and the departure time of the train. Adjustments would obviously have to be made from time-to-time to cater for the demand on various routes. A commuter wishing to use the system indicates his/her choice of ticket (single, return, weekly), origin and destination of travel.

The cost of the ticket and the necessary travel information, such as taxi node/stop, taxi and train departure times are given to the commuter. Upon payment, a printout of the ticket is handed to the passenger. The prototype also allows for a printout of the complete schedule reflecting the integrated timetable, if requested. It is anticipated that a detail schedule will be requested for at the initial stage or commencement of the integrated system until such time commuters become familiar with the timetable. A step-by-step procedure on how to use the prototype is 'built' into the program. Various screens are presented to the commuter which offers the type of ticket, boarding and destination stations, as well as travel information and cost.

It was felt that this article would not have been complete if the financial implications of carrying out a pilot project in the study area were not investigated. The approach considered most logical and appropriate was to look at the two 'extreme' options namely, the integrated ticket and smart card system. It was further agreed to estimate the approximate cost of conducting a pilot project using one of the stations in the area as a test case and allocating a maximum of 50 taxis to the integrated system. The cost of a full-scale pilot project taking into account all five stations and the full commitment of the taxi association in the area could easily be determined/estimated from the 'base' calculation. Taking into account, the hardware, software, and implementation cost of both options, the cost estimate of the ticketing system was just over half that of the smart card system.

\section{CONCLUSION}

An integration technique has been developed in this article based on DFDs in an attempt to integrate the rail with the minibus taxi services. It should be apparent that as powerful as data flow diagrams are, they require further, supporting documents that will define the contents of the data store and defines the processing that takes place in bubbles that have not been partitioned. DFDs are useful throughout the analysis and design process. They can help provide an overview of data movement through the system, lending a visual perspective unavailable in narrative data.

It should be noted that the prototype is to be used as a requirement analysis technique, that is, as a means of determining the client's real need. Thereafter, written specification documents will have to be produced using the prototype as a basis. The technique adopted will make it easier to proceed with the development and refinement of the prototype until it becomes a product. This approach is therefore a viable technique that can lead to fast software development because the knowledge built into the prototype could be easily converted into the final product.

Although the smart card option appears to be much more expensive, this highly versatile electronic system will certainly control fraud and prove to be much more cost effective over the long term.

It would require a multi-dimensional and sustained approach to achieve the target of 80:20 ratio between public transport and private car in the long run as targeted by the government of South Africa. The first step in this direction would be to integrate the existing public transport systems and optimise their usage. An approach to integrate the train and minibus has been suggested in this article and a prototype has been developed for the Chatsworth area in Durban. This integration is likely 
to shift a number of car users towards public transport systems provided other necessary planning and enforcement steps are taken simultaneously. It is recommended that the government take up a few pilot projects to determine the real shift, which will help to identify the measures to be taken to achieve the desired modal split.

\section{REFERENCES}

[1] Black, A., Urban Mass Transportation Planning, McGraw-Hill, Inc., 1995.

[2] Macpherson, G., Highway and Transportation Engineering and Planning, Longman Group UK Limited, 1993.

[3] Department of Transport, White Paper on National Transport Policy, Pretoria, 1996.

[4] Durban Metropolitan Transport Advisory Board, Traffic Monitoring Statistics for the Durban Metropolitan Area, 1995

[5] Traffic and Transportation Department, Port Natal - Ebhodwe: Passenger Transport Plan (Status Quo Report), 1995.

[6] Allopi, D. \& Sarkar, A.K., Assessment on the current transport situation and its impacts on commuter perceptions: a case study. Proceedings of the South African Transport Conference, Vol. 4B, Johannesburg, 1997.

[7] Allopi, D. \& Sarkar, A.K., Increasing the utility of existing transportation infrastructure: a case study. Paper presented at the Third International Conference of the Third World Science, Technology \& Development. Pietermaritzburg, South Africa, 1996.

[8] Allopi, D. \& Sarkar, A.K., Commuter perceptions on private and public transport modes: a case study. Proceedings of the Third International Conference on Urban Transport and the Environment for the 21st Century, Computational Mechanics: Southampton, UK, 1997.

[9] Powers, M.J, Cheney, P.H. \& Crow, G., Structured Systems Development Analysis, Design, Implementation, 2nd edn, Boyd \& Fraser, 1990.

[10] Allopi, D. \& Sarkar, A.K., The integration of minibus and rail services. Journal of the South African Institution of Civil Engineering, 4(2), pp. 22-26, 2001. 